\title{
An Approximate Optimal Maximum Range Guidance Scheme for Subsonic Unpowered Gliding Vehicles
}

\author{
Dao-Chi Zhang, Qun-Li Xia, Qiu-Qiu Wen, and Guan-qun Zhou \\ School of Aerospace Engineering, Beijing Institute of Technology, Beijing 100081, China \\ Correspondence should be addressed to Qiu-Qiu Wen; wenqiuqiu82@bit.edu.cn
}

Received 30 July 2015; Revised 1 November 2015; Accepted 8 November 2015

Academic Editor: Paul Williams

Copyright ( 2015 Dao-Chi Zhang et al. This is an open access article distributed under the Creative Commons Attribution License, which permits unrestricted use, distribution, and reproduction in any medium, provided the original work is properly cited.

\begin{abstract}
This study investigates the maximum gliding range problems of subsonic unpowered gliding vehicles and proposes an approximate optimal maximum range guidance scheme. First, the gliding flight path angle corresponding to constant dynamic pressure is derived. A lift-to-drag ratio $(L / D)$ inversely proportional to the dynamic pressure is then proven. On this basis, the calculation method of an optimal dynamic pressure (ODP) profile with a maximum $L / D$ throughout the flight is presented. A guidance scheme for tracking the ODP profile, which uses the flight path angle as control variable, is then designed. The maximum ranges of the unpowered gliding vehicle obtained by the proposed guidance scheme and pseudospectral method are compared. Results show that the guidance scheme provides an accurate approximation of the optimal results, and the errors are less than $2 \%$. The proposed guidance scheme is easy to implement and is not influenced by wind compared with numerical schemes.
\end{abstract}

\section{Introduction}

Identifying the maximum range and an optimal guidance scheme is extremely important for unpowered gliding vehicles, which possess a limited amount of initial energy. For example, every airplane or unmanned aerial vehicle becomes a glider when the engine fails (power off); thus, determining the maximum reachable domain, selecting a proper landing site, and proposing a feasible trajectory toward the site for flight management are important [1]. The problems of maximum range trajectories and an optimal guidance scheme of subsonic unpowered gliding vehicles have been extensively studied for decades. The related methods can be divided into three categories, namely, trajectory optimization method, singular perturbation method, and quasi-steady approximation.

The problem of designing maximum range trajectories can be considered a trajectory optimization problem. The processes for optimizing trajectories fall into two broad approaches, namely, indirect and direct methods. The indirect method is based on Pontryagin's minimum modulus principle and transforms an optimal control problem to a
Hamiltonian boundary value problem. This method has the advantages of high accuracy and satisfies first-order necessary conditions. Vinh et al. [2] obtained a solution for the optimal subsonic gliding flight of an unpowered gliding vehicle in horizontal plane by applying Pontryagin's maximum principle. Lu [3] studied the problems of optimal entry trajectories by using an indirect method and obtained a closed-form, near-optimal bank angle control law for lifting entry vehicles. However, the indirect method has the disadvantages of complicated calculation and hypersensitivity to initial value, which limit its applicability.

The direct method first transforms a continuous optimal control problem to a nonlinear programming problem by dispersing and parameterizing the control and state variables and then resolving the problem by a numerical method. Betts [4] and Huang et al. [5] provided excellent reviews and surveys of trajectory optimization numerical methods. The direct method does not need the necessary condition to resolve an optimal solution and can incorporate any trajectory constraints and any level of modeling fidelity [3]. With the development of the computer technology, the direct method has been widely used to calculate optimal 
reference trajectories with multiple constraints [6-8]. The pseudospectral method [9-12] (or orthogonal collocation method), as a new direct numerical method for optimal problems, has attracted considerable interest in recent years. This method uses global polynomial approximation state and control variables and has the characteristics of minimal parameters, high accuracy, fast convergence, and insensitivity to initial value. This method is widely used in aircraft trajectory optimization problems $[13,14]$. A typical research result of the pseudospectral method is the famous optimal problem-solving software GPOPS, which was developed by Rao's research team. This software is used in the current study to calculate numerical optimal solutions. However, the direct method obtains an open-loop guidance scheme and has the reliability of convergence and computation efficiency; it is difficult to directly implement in a real-time system.

Singular perturbation theory, as an approximate solution to a differential equation, is extensively used in solving the optimal trajectory problem. Sheu $[15,16]$ studied the optimal gliding problem by using singular perturbation methods and obtained a closed-loop online optimal feedback guidance law. Shapira $[17,18]$ studied the optimal gliding problem by using different timescales and indicated that timescale separation affected the optimal results. Naidu and Calise [19] provided an overview of singular perturbation methods for guidance and control problems. Although it can produce analytical closed-loop laws with a minimal sacrifice of accuracy, the singular perturbation method also needs numerous calculations and is simplified in deviation; hence, it is unsuitable for the real-time control of a vehicle.

The highly nonlinear equation of motion and complex aerodynamic models prevent the maximum gliding problem from generating analytic solutions. Several scholars have simplified analysis models based on quasi-steady approximates and designed a near-optimal gliding scheme. Kelley et al. [20] proved that the maximum lift-to-drag ratio $\left(L / D_{\max }\right)$ operation is an approximately optimal glide range through a variable-density atmosphere in a flat Earth based on quasisteady approximation and proposed a scheme to control gliding vehicles. Phillips [21] used the scheme to expand the range of guided projectiles. Yu and Chen [22] proposed a guidance scheme that controlled the flight close to $L / D_{\max }$ with flight path angle feedback to dampen phugoid oscillation. Indig et al. [23] designed a near-optimal guidance law on the basis of the result of numerical optimal trajectories obtained by GPOPS. De Ridder [24, 25] demonstrated the connection between minimum drag and maximum range trajectories and developed an approximate maximum range TAEM guidance algorithm for unpowered RLV by using the angle of attack as a control variable.

The contribution of the present study is to provide an approximate optimal gliding guidance scheme for subsonic unpowered gliding vehicles. Unlike prior works, the current guidance scheme does not need numerous computations. The guidance scheme is based on $L / D_{\max }$ flight but converts $L / D_{\max }$ flight to track the optimal dynamic profile varying with height. The optimal dynamic profile only relates to vehicle shape and only needs to calculate once for a specific unpowered gliding vehicle. A guidance scheme using flight path angle as control variable is also designed, and the flight path angle command is calculated in real time to track the optimal dynamic pressure (ODP) profile. The simulation results indicate that the proposed guidance scheme accurately approximates the optimal results, which are obtained by an hp-adaptive pseudospectral method using GPOPS. Compared with the numerical method, the proposed guidance scheme has the benefits of being easy to implement, can adapt to all initial states, and is not influenced by wind and atmospheric model variations.

\section{ODP Profile with $L / D_{\max }$}

For the performance prediction of the maximum range, a point mass model for motion in a vertical plane is usually adequate [24]. The equations of motion are expressed as follows:

$$
\begin{aligned}
& \dot{V}=-\frac{\left(C_{D} q S+m g \sin \gamma\right)}{m}, \\
& \dot{\gamma}=\frac{\left(C_{L} q S-m g \cos \gamma\right)}{m V}, \\
& \dot{R}=V \cos \gamma, \\
& \dot{H}=V \sin \gamma,
\end{aligned}
$$

where $H$ is the altitude, $R$ is the gliding range, $V$ is the speed, $\gamma$ is the flight path angle, $C_{L}$ and $C_{D}$ are the lift and drag coefficients, respectively, and $q, S, m$, and $g$ are the flight dynamic pressure, reference area, vehicle mass, and gravitational acceleration, respectively.

The energy height $E$ is defined as the total energy divided by weight as follows:

$$
E=\frac{V^{2}}{2 g}+H .
$$

Reference [20] proved that gliding range can be calculated by the following:

$$
\Delta R=\int_{E_{\mathrm{FIN}}}^{E_{\mathrm{INI}}} \frac{C_{L}}{C_{D}} d E,
$$

where $\Delta R$ is the flight range. $E_{\mathrm{INI}}$ and $E_{\mathrm{FIN}}$ are the initial and finial energy heights, respectively.

The proven process can be found in [20]. From (6), the gliding range can be maximized when $L / D$ is maximized throughout the flight. If $C_{L}$ and $C_{D}$ are approximately constant with Mach number and flight path angle, $L / D_{\max }$ flight is at a constant dynamic pressure.

The definition of dynamic pressure is expressed as follows:

$$
q=\frac{1}{2} \rho V^{2}
$$

where $q$ is the dynamic pressure and $\rho$ is the air density. For analytical reasons, an exponential approximation of atmospheric density is used:

$$
\rho=\rho_{0} e^{\beta_{0} H}
$$

where $\rho_{0}$ and $\beta_{0}$ are the constants. 
Differentiating (7) with respect to $t$ yields the following:

$$
\dot{q}=\frac{1}{2} \rho_{0} \beta_{0} \dot{h} e^{\beta_{0} h} V^{2}+\rho_{0} e^{\beta_{0} h} V \dot{V} .
$$

The dynamic pressure is kept constant, so $\dot{q}=0$. Substituting (1) and (7) into (9) after some algebraic calculations yields the following:

$$
\sin \gamma=\frac{C_{D} q S \rho_{0} e^{\beta_{0} h}}{m\left(q \beta_{0}-g \rho_{0} e^{\beta_{0} h}\right)} .
$$

Substituting (8) into (10) yields the following:

$$
\gamma_{\mathrm{CDP}}=a \sin \left[\frac{C_{D} q S \rho}{m\left(q \beta_{0}-g \rho\right)}\right]
$$

where $\gamma_{\mathrm{CDP}}$ is the gliding path angle corresponding to constant dynamic pressure. From (11), $\gamma_{\mathrm{CDP}}$ is related to dynamic pressure, vehicle drag coefficient, reference area, vehicle mass, and atmospheric density.

Differentiating (10) with respect to $\gamma$ and after simplification yields the following:

$$
\dot{\gamma}=\frac{q \beta_{0}{ }^{2} C_{d} q S \rho \dot{h}}{m \sqrt{1-\sin ^{2} \gamma}\left(q \beta_{0}-g \rho\right)^{2}} .
$$

After substituting (2) into (12) and after a complex operation, the relationship between dynamic pressure and $L / D$ is obtained as follows:

$$
\frac{C_{L}}{C_{D}}=\frac{g\left[m^{2} A^{2}-D^{2} \rho^{2}\right] A+2 q^{2} \beta_{0}^{2} D^{2} \rho}{D A^{2} \sqrt{m^{2} A^{2}-D^{2} \rho^{2}}},
$$

where

$$
\begin{aligned}
& A=q \beta_{0}-g \rho, \\
& D=C_{D} q S .
\end{aligned}
$$

From (13), for a specific flight vehicle, the gliding $L / D$ with a constant dynamic pressure only relates to dynamic pressure and drag coefficient. The relationship between $L / D$ and dynamic pressure according to (13) is shown in Figure 1, with various drag coefficients. As shown in Figure 1, $L / D$ is inversely proportional to constant dynamic pressure. A higher dynamic pressure leads to a smaller gliding $L / D$.

For a specified gliding vehicle, $L / D_{\max }$ and corresponding drag coefficient can be calculated from the aerodynamic data. If the aerodynamic coefficients remain constant with the Mach number, the ODP with $L / D_{\max }$ can be calculated by the Newton-Raphson method by using (13). If the Mach number has an effect on the aerodynamic coefficients, the ODP profile with $L / D_{\max }$ throughout the flight can be calculated by using the equation stated above in an iteration process. The required steps to calculate a single point on the dynamic profile are shown in Figure 2. This process is repeated for all flight heights, and the $L / D_{\max }$ dynamic pressure profile varying with height can be obtained.

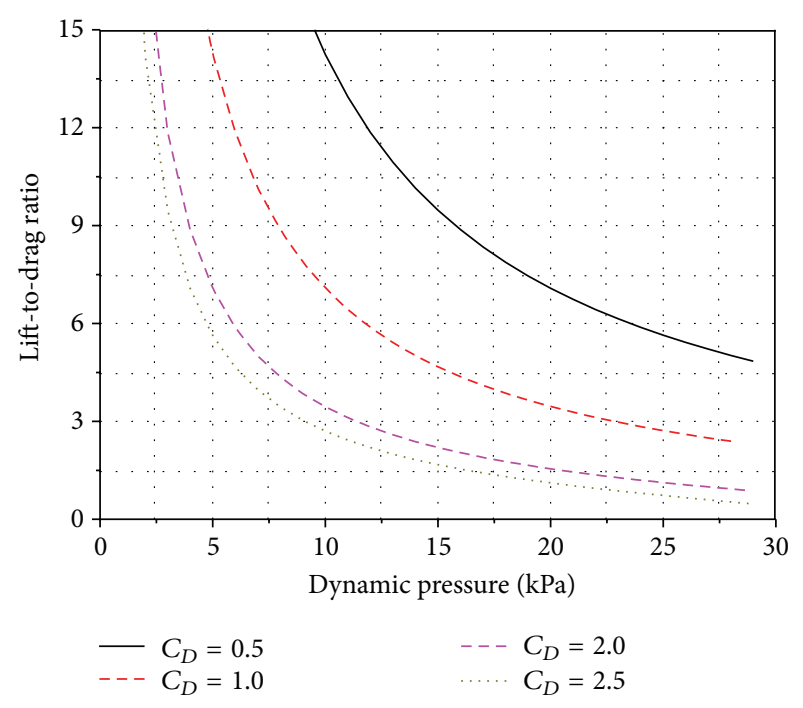

FIGURE 1: Relations between dynamic pressure and lift-to-drag ratio $(L / D)$.

\section{Maximum Range Guidance Scheme Design}

As aforementioned, gliding at $L / D_{\max }$ during flight is an optimal strategy for a maximum range, which can be equivalent to flight at a constant ODP or an ODP profile with $L / D_{\max }$.

From (11), a constant dynamic pressure flight can be transformed to maintain a certain flight path angle. Hence, we use flight path angle as the variable to control flight dynamic pressure. Considering that the initial dynamic pressure may be not equal to the optimal value, the flight path angle command is expressed as follows:

$$
\gamma_{c}=\gamma_{\mathrm{ODP}}+\Delta \gamma
$$

where $\gamma_{\mathrm{ODP}}$ is the flight path angle corresponding to ODP, which is calculated by (11). $\Delta \gamma$ is the compensated flight path angle, which regulates flight dynamic pressure or compensates an uncertain error and a steady-state tracking error. $\Delta \gamma$ is calculated as follows:

$$
\Delta \gamma=k_{q}\left(q_{\mathrm{ODP}}-q\right),
$$

where $k_{q}$ is the dynamic pressure adjustment coefficient, $q_{\mathrm{ODP}}$ is the ODP with $L / D_{\max }$, and $q$ is the flight dynamic pressure.

$k_{q}$ is induced to adjust the initial flight dynamic pressure to an optimal value and has an effect on the initial trajectory. The value of $k_{q}$ can be selected by approximating the optimal trajectory obtained by optimal control theory or the numerical results.

\section{Simulation Results}

In this section, the simulation of an unpowered gliding vehicle is used to verify the guidance scheme. The results are compared with the optimal results obtained by a pseudospectral method using GPOPS V5.0. See [26] for the method and its applications. 
TABle 1: $L / D_{\max }$ and drag coefficient in different Mach numbers.

\begin{tabular}{lcc}
\hline Mach number & $L / D_{\max }$ & Drag coefficient CD \\
\hline 0.4 & 7.84 & 0.343 \\
0.6 & 8.29 & 0.337 \\
0.8 & 8.22 & 0.378 \\
0.9 & 5.78 & 0.561 \\
1.0 & 4.28 & 1.0 \\
\hline
\end{tabular}

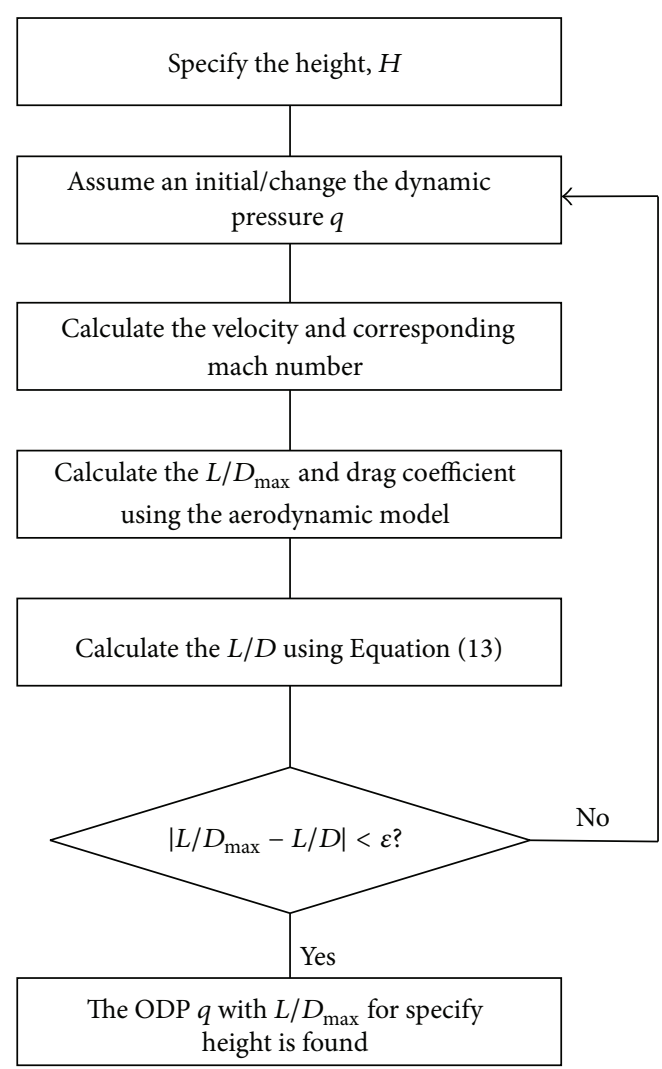

FIGURE 2: Steps for calculating dynamic pressure with $L / D_{\max }$ for a specified flight height.

4.1. Simulation Model. Equations (1)-(4) are used as unpowered gliding vehicle motion models where the mass of the gliding vehicle is $500 \mathrm{~kg}$ and the reference area is $0.25 \mathrm{~m}^{2} . L / D$ and drag coefficient with different Mach numbers are shown in Figure 3 and Table 1.

The used atmosphere model is the 1976 US standard atmosphere. One of the air density approximations for the 1976 US standard atmosphere is given by the following:

$$
\rho= \begin{cases}1.232 e^{-0.1024 H}, & H \leq 6 \\ 1.374 e^{-0.1207 H}, & 6<H \leq 11 \\ 2.059 e^{-0.1572 H}, & H>11\end{cases}
$$

where $H$ is the altitude and is measured in kilometer.

The ODP profile that varies with flight height is calculated by the method mentioned above and shown in Figure 4. When the height is less than $3600 \mathrm{~m}$, the flight Mach number is less than 0.4 and the aerodynamic coefficient is not
TABLE 2: Suboptimal gains for the maximum range $\Delta q=\left|q_{\mathrm{ref}}-q\right|$.

\begin{tabular}{lc}
\hline$\Delta q, \mathrm{~Pa}$ & $k_{q}, \mathrm{deg} . / \mathrm{Pa}$ \\
\hline$>5000$ & 0.003 \\
2500 & 0.006 \\
$<500$ & 0.01 \\
\hline
\end{tabular}

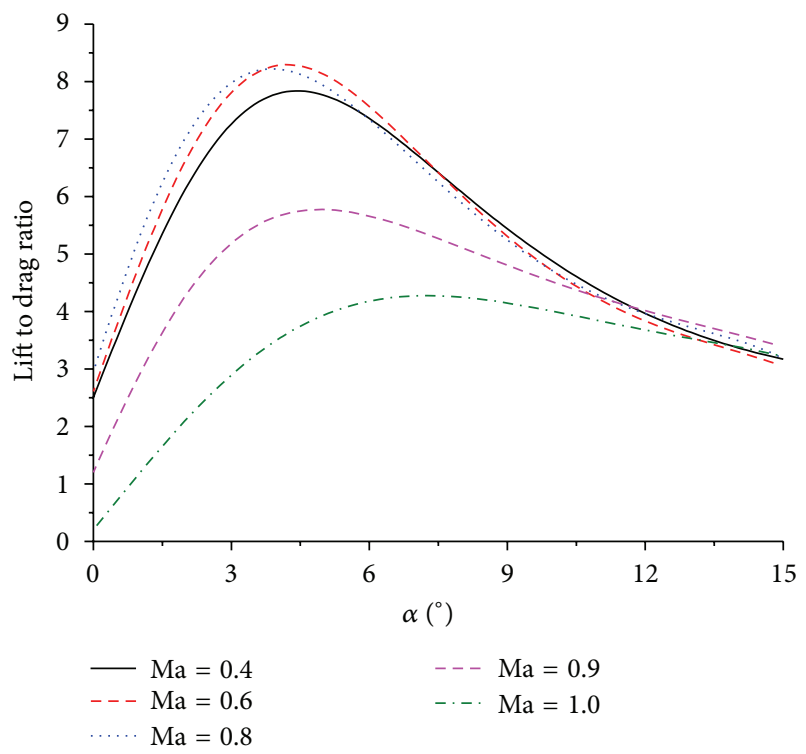

Figure 3: $L / D$ versus angle of attack.

correlated with the Mach number. Consequently, the ODP is constant.

The state equations used in the pseudospectral method are (1)-(4). The control variable is $\alpha$. For a specific height, the gliding terminals $\gamma$ and $q_{f}$ are definite by using the proposed guidance scheme and these terminal conditions are used as boundary constraints in the pseudospectral method for the same initial and terminal conditions with two methods. The terminal gliding height is $3 \mathrm{~km}$. Other boundary constraints are as follows:

$$
\begin{aligned}
\alpha & \in\left(-15^{\circ}, 15^{\circ}\right), \\
\dot{\alpha} & \in\left(-3^{\circ} / \mathrm{s}, 3^{\circ} / \mathrm{s}\right), \\
H_{f} & =3 \mathrm{~km}, \\
q_{f} & =7200 \mathrm{~Pa}, \\
\gamma_{f} & =-6.7^{\circ} .
\end{aligned}
$$

The cost function is expressed as follows:

$$
J=-R \text {. }
$$

Initial conditions with different initial dynamic pressures are used to design a suboptimal value of dynamic pressure adjustment gain $k_{q}$. Table 2 presents the obtained suboptimal values of gains. If the initial dynamic pressure falls into $[500,2500]$ or $[2500,5000]$, the gain is calculated by the $1 \mathrm{D}$ linear interpolation with dynamic pressure; otherwise, the gain is constant. 
TABLE 3: Initial conditions and obtained maximum range using the proposed guidance scheme and optimal method.

\begin{tabular}{lccccccccc}
\hline \multirow{2}{*}{ Scenario } & \multirow{2}{*}{$H(\mathrm{~km})$} & $V(\mathrm{~m} / \mathrm{s})$ & \multirow{2}{*}{$E(\mathrm{~km})$} & \multicolumn{2}{c}{ Initial } & \multicolumn{2}{c}{ Proposed } & \multicolumn{3}{c}{ Optimal } \\
& & & & $q-q_{\mathrm{ref}}$ & $R(\mathrm{~km})$ & $R_{\mathrm{ae}}$ & $R(\mathrm{~km})$ & $R_{\mathrm{ae}}$ & \multicolumn{2}{c}{ Error $(\%)$} \\
\hline 1 & 9.0 & 175 & 10.56 & 0 & 51.33 & 7.60 & 51.84 & 7.68 & 0.98 \\
2 & 8.0 & 224 & 10.56 & 5826 & 50.89 & 7.54 & 51.34 & 7.61 & 0.87 \\
3 & 10.0 & 290 & 14.29 & 10392 & 78.18 & 7.46 & 79.08 & 7.54 & 1.14 \\
4 & 6.0 & 299 & 10.56 & 22320 & 44.78 & 6.63 & 45.39 & 6.72 & 1.3 \\
5 & 10.0 & 105 & 10.56 & -4618 & 49.85 & 7.39 & 50.68 & 7.51 & 1.63 \\
6 & 8.0 & 140 & 9.00 & -2171 & 38.78 & 7.47 & 39.32 & 7.58 & 1.38 \\
\hline
\end{tabular}

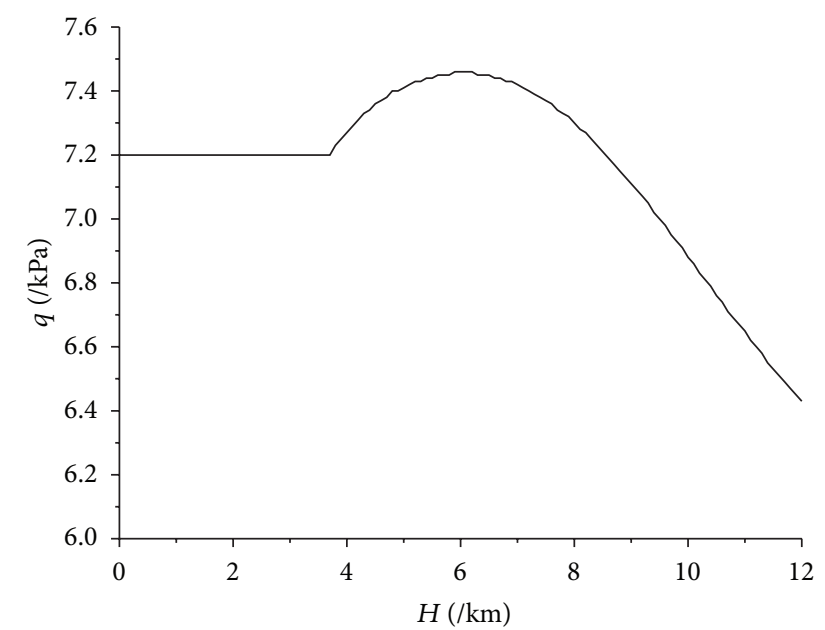

Figure 4: ODP profile.

\subsection{Simulation Results}

4.2.1. Nominal Simulation Result. The initial conditions and obtained maximum ranges obtained by the proposed scheme and pseudospectral method are shown in Table 3. The initial flight path angle for all conditions is $0^{\circ}$. From the table, all the obtained ranges are $2 \%$ less than the optimal results, which are accurate approximations to the optimal ranges.

To evaluate the gliding capability in different initial conditions, we define $R_{\mathrm{ae}}$ as the average gliding range by unit energy height as follows:

$$
R_{\mathrm{ae}}=\frac{\Delta R}{\Delta E}
$$

where $\Delta E=E_{\text {Ini }}-E_{\text {Fin }}$ and $\Delta R$ is the gliding range.

$R_{\mathrm{ae}}$ represents the gliding efficiency of the unpowered gliding vehicle. A large $R_{\mathrm{ae}}$ value indicates high gliding capability. Table 2 shows that Scenarios 1, 2, 4, and 5 have the same initial energy height with different distributions of kinetic and potential energies. The maximum range differs because the dynamic pressure adjustment to an optimal value needs to consume energy. If the initial dynamic pressure is equal to the optimal value, the range reaches the maximum value (Scenario 1). The results imply that a larger initial $\mid q_{\text {ref }}-$ $q$ | denotes a smaller $R_{\mathrm{ae}}$. For all results, $R_{\mathrm{ae}}$ is almost the same. Therefore, the best gliding efficiency is fixed for a specified flight vehicle, and the proposed guidance scheme achieves the best gliding efficiency for different initial states.
TABLE 4: Wind velocity in different altitudes.

\begin{tabular}{lccc}
\hline Altitude $(\mathrm{km})$ & 0.0 & 3.0 & 10.0 \\
\hline Wind velocity $(\mathrm{m} / \mathrm{s})$ & 10.0 & 20.0 & 40.0 \\
\hline
\end{tabular}

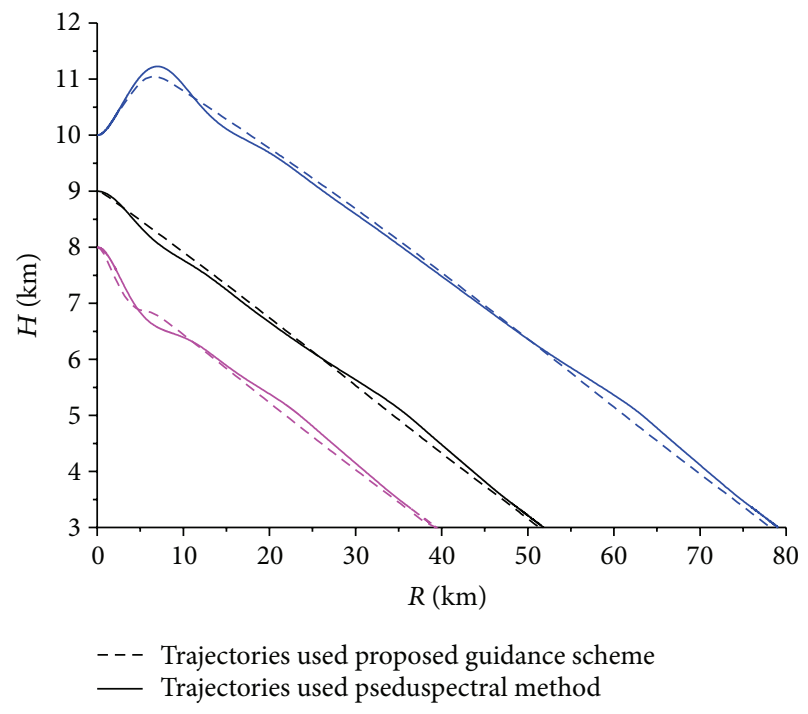

FIGURE 5: Gliding trajectories for Scenarios 1, 3, and 6.

The trajectories, flight path angles, and velocities (Scenarios 1, 3, and 6) obtained by the proposed guidance scheme and pseudospectral method are shown in Figures 5-7. From these trajectories, the proposed guidance scheme eliminates oscillation in contrast to the optimal result. The proposed guidance scheme trajectory is close to the optimal one and has a good performance. The $L / D$ and $L / D_{\max }$ curves during flight are shown in Figure 8. The figure shows that the proposed guidance scheme follows $L / D_{\max }$.

The initial states with an altitude between 6.0 and $10.0 \mathrm{~km}$ and a velocity between 100.0 and $300 \mathrm{~m} / \mathrm{s}$ are calculated by using the gains in Table 2 to verify the sub-ODP adjustment gain. The gliding efficiency $R_{\mathrm{ae}}$ for all initial states is shown in Figure 9. The figure confirms that the guidance scheme is valid for all initial states.

\subsubsection{Simulation Results Considering Variation}

(a) Wind Disturbance. The wind velocity in the wind disturbance simulation is presented in Table 4 . The wind 
TABLE 5: Simulation results with wind disturbance.

\begin{tabular}{|c|c|c|c|c|c|c|c|c|c|}
\hline \multirow[b]{2}{*}{ Scenario } & \multirow[b]{2}{*}{$H(\mathrm{~km})$} & \multirow[b]{2}{*}{$V(\mathrm{~m} / \mathrm{s})$} & \multirow[b]{2}{*}{ Wind direction } & \multirow[b]{2}{*}{ Terminal $E(\mathrm{~km})$} & \multicolumn{2}{|c|}{ Proposed } & \multicolumn{2}{|c|}{ Optimal } & \multirow[b]{2}{*}{ Error $(\%)$} \\
\hline & & & & & $\begin{array}{c}\text { Range } \\
(\mathrm{km})\end{array}$ & $\begin{array}{c}R_{\mathrm{ae}} \\
(\mathrm{km})\end{array}$ & $\begin{array}{c}\text { Range } \\
(\mathrm{km})\end{array}$ & $\begin{array}{l}R_{\mathrm{ae}} \\
(\mathrm{km})\end{array}$ & \\
\hline 7 & 9.0 & 175.0 & Against the wind & 3.57 & 44.42 & 6.35 & 45.19 & 6.46 & 1.70 \\
\hline 8 & 9.0 & 175.0 & Following the wind & 4.09 & 56.53 & 8.73 & 58.19 & 8.99 & 2.85 \\
\hline
\end{tabular}

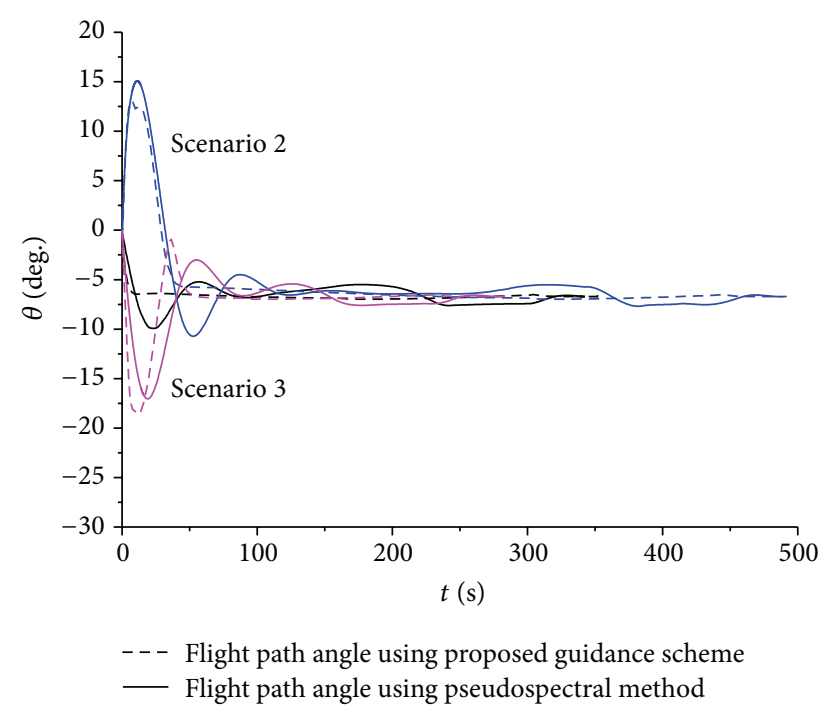

FIGURE 6: Flight path angles for Scenarios 1, 3, and 6.

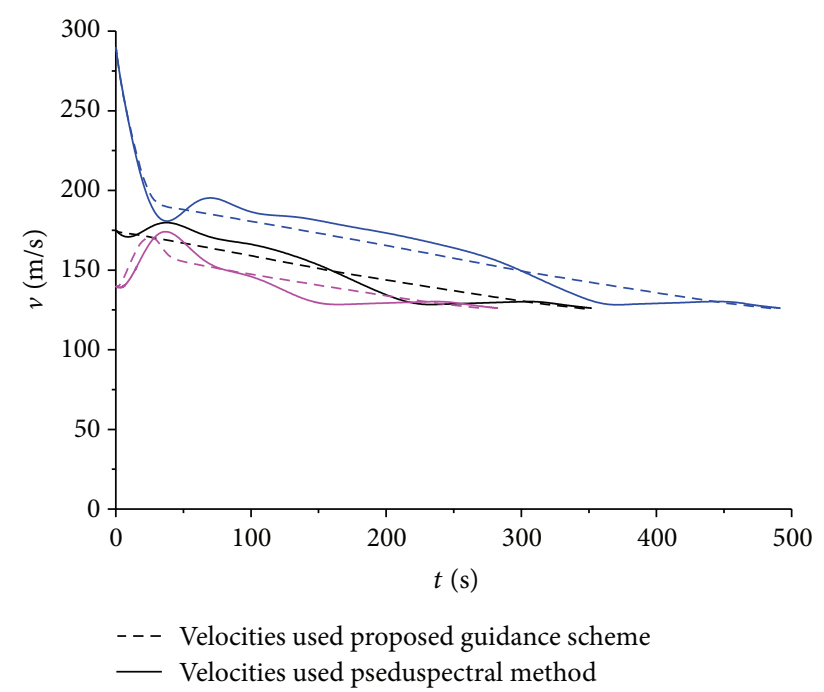

Figure 7: Velocities for Scenarios 1, 3, and 6.

velocity is calculated by one-dimensional linear interpolation with altitude when the flight height falls into $(0,3000)$ or $(3000,10000)$. When the height is larger than $10 \mathrm{~km}$, the wind velocity remains constant, and the value is $40 \mathrm{~m} / \mathrm{s}$.

Table 5 and Figure 10 show the results and trajectories obtained by the proposed guidance scheme and the optimal results. From the results, when the vehicle flies following wind, it gets a longer range than in the no-wind condition;

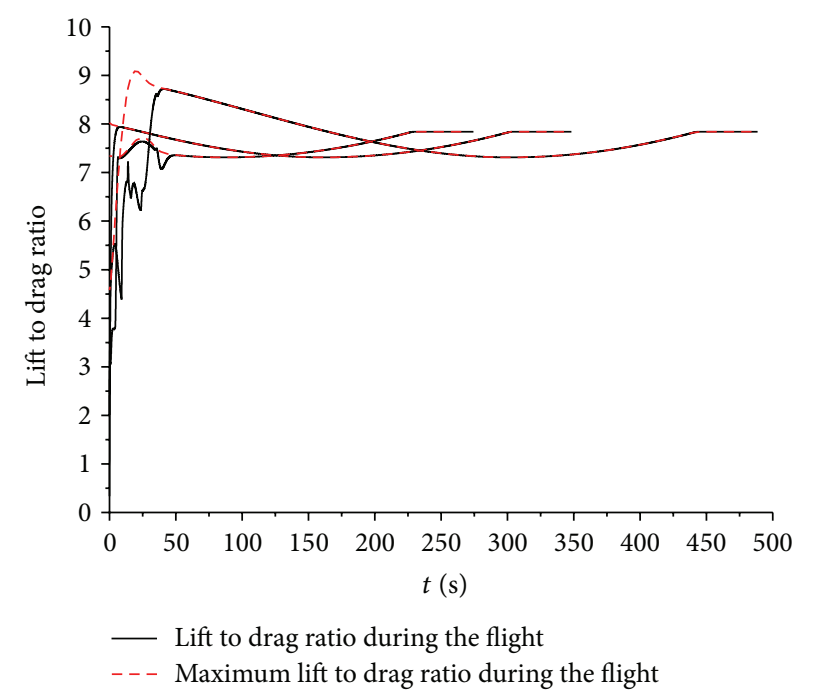

Figure 8: $L / D$ and corresponding $L / D_{\max }$ for Scenarios 1,3 , and 6.

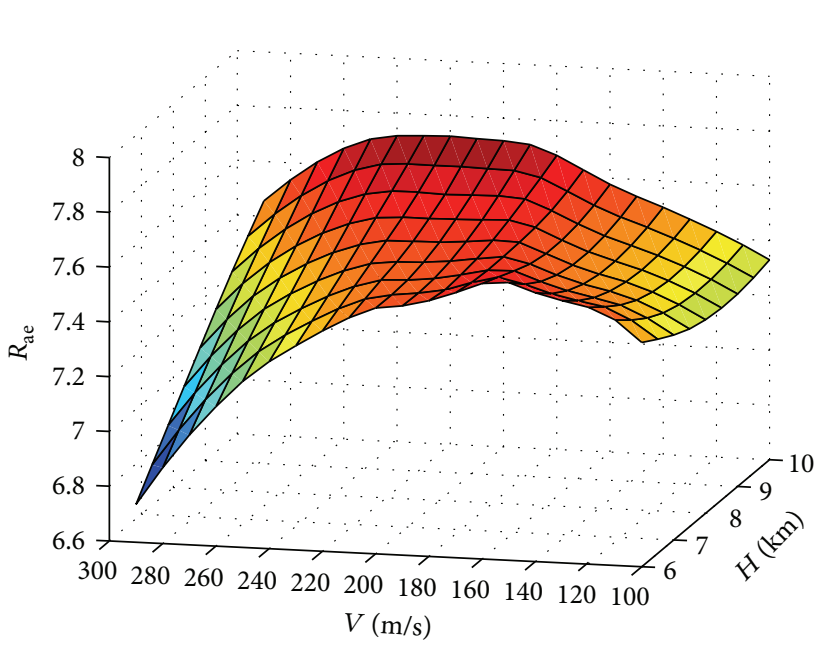

FIGURE 9: $R_{\mathrm{ae}}$ with different initial states.

when it flies against the wind, the gliding range is smaller. Flying against the wind increases drag, whereas following the wind leads to the contrary effect. From $R_{\mathrm{ae}}$, following and flying against the wind increase and decrease gliding efficacy, respectively. We can also determine that the guidance scheme is close to optimal results and is valid in wind disturbance.

(b) Atmospheric Variations. The atmospheric model that varies from the assumption in the derivation is considered. 
TABLE 6: Simulation results with atmospheric variations.

\begin{tabular}{lccccccccc}
\hline Scenario & $H(\mathrm{~km})$ & $V(\mathrm{~m} / \mathrm{s})$ & Atmospheric variation & Terminal $E(\mathrm{~km})$ & \multicolumn{2}{c}{ Proposed } & \multicolumn{3}{c}{ Optimal } \\
& & & & & $(\mathrm{km})$ & $\begin{array}{c}R_{\mathrm{ae}} \\
(\mathrm{km})\end{array}$ & $\begin{array}{c}\text { Range } \\
(\mathrm{km})\end{array}$ & $\begin{array}{c}R_{\mathrm{ae}} \\
(\mathrm{km})\end{array}$ & $\begin{array}{c}\text { Error }(\%) \\
10\end{array}$ \\
\hline 9 & 9.0 & 175.0 & $+5 \%$ & 3.77 & 51.62 & 7.60 & 52.12 & 7.68 & 0.96 \\
10 & 9.0 & 175.0 & $+10 \%$ & 3.73 & 51.81 & 7.59 & 52.37 & 7.67 & 1.10 \\
11 & 9.0 & 175.0 & $-5 \%$ & 3.85 & 51.00 & 7.60 & 51.61 & 7.69 & 1.18 \\
12 & 9.0 & 175.0 & $-10 \%$ & 3.89 & 50.67 & 7.60 & 51.35 & 7.70 & 1.32 \\
\hline
\end{tabular}

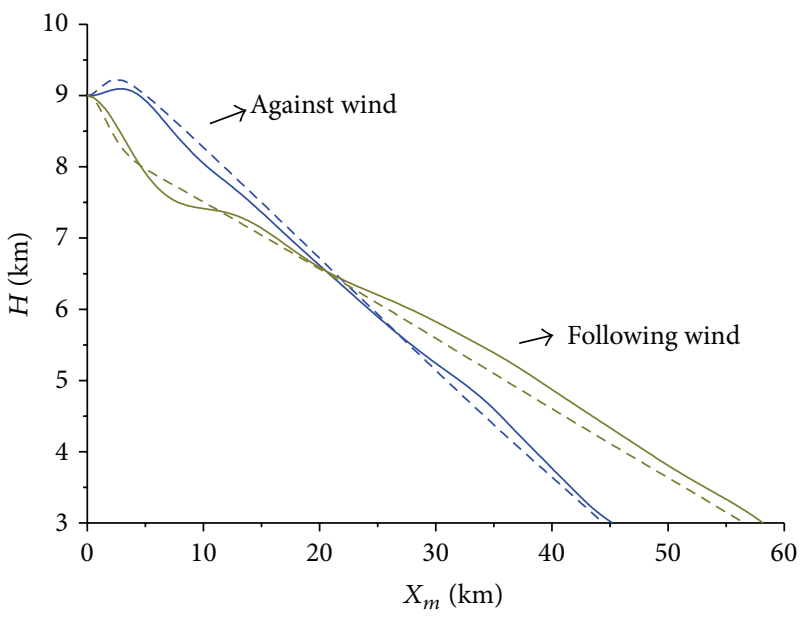

_ Optimal trajectories obtained by pseudospectral method - - - Trajectories obtained by proposed guidance scheme

Figure 10: Gliding trajectories with wind disturbance.

In this case, atmospheric density is increased or decreased as a fixed percentage of value from the nominal atmospheric model.

Table 6 summarizes the atmospheric variation results. In all cases, the initial states are fixed. Compared with Scenario 1, atmospheric density increases or decreases by $10 \%$, the range increases or decreases by only $1 \%$, and $R_{\mathrm{ae}}$ is the same when atmospheric density varies. Thus, atmospheric variation has insignificant effects on gliding efficiency.

\section{Conclusions}

A new approximate optimal gliding scheme for subsonic unpowered gliding vehicles is presented. The relationship between $L / D$ and dynamic pressure is derived. On this basis, a calculation method that computes the variations of the ODP profile with flight height, which corresponds to $L / D_{\max }$, is proposed. For a specific vehicle, the ODP profile is constant. By using flight path angle as a control variable, a guidance scheme is designed to control the vehicle to track the ODP profile. Simulations indicate that the proposed scheme accurately approximates the optimal ranges for all initial states and is not influenced by wind and atmospheric model variations.

\section{Conflict of Interests}

The authors declare no conflict of interests.

\section{References}

[1] H. Wu and F. Mora-Camino, "Glide control for engine-out aircraft," in Proceedings of the AIAA Guidance, Navigation, and Control Conference (GNC '12), Minneapolis, Minn, USA, August 2012.

[2] N. X. Vinh, C.-Y. Yang, and J.-S. Chern, "Optimal trajectories for maximum endurance gliding in a horizontal plane," Journal of Guidance, Control, and Dynamics, vol. 7, no. 2, pp. 246-248, 1984.

[3] P. Lu, "Entry trajectory optimization with analytical feedback bank angle law," in Proceedings of the AIAA Guidance, Navigation and Control Conference and Exhibit, AIAA 2008-7268, Honolulu, Hawaii, USA, April 2008.

[4] J. T. Betts, "Survey of numerical methods for trajectory optimization," Journal of Guidance, Control, and Dynamics, vol. 21, no. 2, pp. 193-207, 1998.

[5] G. Huang, Y. Lu, and Y. Nan, "A survey of numerical algorithms for trajectory optimization of flight vehicles," Science China Technological Sciences, vol. 55, no. 9, pp. 2538-2560, 2012.

[6] L. H. Tu, J. P. Yuan, Q. Fang, and J. J. Luo, "Reentry skipping trajectory optimization using direct parameter optimization method," in Proceedings of the 14th AIAA/AHI Space Planes and Hypersonic Systems and Technologies Conference, p. 7993, Canberra, Australia, 2006.

[7] P. J. Enright and B. A. Conway, "Discrete approximations to optimal trajectories using direct transcription and nonlinear programming," Journal of Guidance, Control, and Dynamics, vol. 15, no. 4, pp. 994-1002, 1992.

[8] P. Lu, "Inverse dynamics approach to trajectory optimization for an aerospace plane," Journal of Guidance, Control, and Dynamics, vol. 16, no. 4, pp. 726-732, 1993.

[9] F. Fahroo and I. M. Ross, "Direct trajectory optimization by a Chebyshev pseudospectral method," Journal of Guidance, Control, and Dynamics, vol. 25, no. 1, pp. 160-166, 2002.

[10] D. Benson, A Gauss pseudospectral transcription for optimal control [Ph.D. thesis], Massachusetts Institute of Technology, Cambridge, Mass, USA, 2005.

[11] G. T. Huntington, D. Benson, and A. V. Rao, "A comparison of accuracy and computational efficiency of three pseudospectral methods," in Proceedings of the AIAA Guidance, Navigation and Control Conference, Paper no. AIAA 2007-6405, pp. 840-864, Hilton Head Island, SC, USA, August 2007.

[12] D. A. Benson, G. T. Huntington, T. P. Thorvaldsen, and A. V. Rao, "Direct trajectory optimization and costate estimation 
via an orthogonal collocation method," Journal of Guidance, Control, and Dynamics, vol. 29, no. 6, pp. 1435-1440, 2006.

[13] C. C. Françolin and A. V. Rao, "Direct trajectory optimization and costate estimation of state inequality path-constrained optimal control problems using a radau pseudospectral method," in Proceedings of the AIAA Guidance, Navigation, and Control Conference, Minneapolis, Minn, USA, August 2012.

[14] J. Rea, "Launch vehicle trajectory optimization using a Legendre pseudospectral method," in Proceedings of the AIAA Guidance, Navigation and Control Conference, Paper no. AIAA 2003-5640, Austin, Tex, USA, August 2003.

[15] D. Sheu, Y.-M. Chen, Y.-J. Chang, and J.-S. Chern, "Optimal glide for maximum range," in Proceedings of the AIAA Atmospheric Flight Mechanics Conference and Exhibit, pp. 771-781, Boston, Mass, USA, 1998.

[16] D. Sheu, Y.-M. Chen, and J.-S. Chern, "Optimal threedimensional glide for maximum reachable domain," in Proceedings of the 24th AIAA Atmospheric Flight Mechanics Conference, AIAA, Portland, Ore, USA, August 1999.

[17] I. Shapira and J. Z. Ben-Asher, "Singular perturbation analysis of optimal glide," Journal of Guidance, Control, and Dynamics, vol. 27, no. 5, pp. 915-918, 2004.

[18] I. Shapira and J. Ben-Asher, "Range maximization for emergency landing after engine cutoff," Journal of Aircraft, vol. 42, no. 5, pp. 1296-1306, 2005.

[19] D. S. Naidu and A. J. Calise, "Singular perturbations and time scales in guidance and control of aerospace systems: a survey," Journal of Guidance, Control, and Dynamics, vol. 24, no. 6, pp. 1057-1078, 2001.

[20] H. J. Kelley, E. M. Cliff, and F. H. Lutze, "Boost-glide rangeoptimal guidance," in Guidance and Control Conference, American Institute of Aeronautics and Astronautics, 1981.

[21] C. A. Phillips, "Guidance algorithm for range maximization and time-of-flight control of a guided projectile," Journal of Guidance, Control, and Dynamics, vol. 31, no. 5, pp. 1447-1455, 2008.

[22] W. Yu and W. Chen, "Guidance scheme for glide range maximization of a hypersonic vehicle," in Proceedings of the AIAA Guidance, Navigation, and Control Conference, vol. 6714, Portland, Ore, USA, August 2011.

[23] N. Indig, J. Z. Ben-Asher, and N. Farber, "Near-optimal spatial midcourse guidance law with an angular constraint," Journal of Guidance, Control, and Dynamics, vol. 37, no. 1, pp. 214-223, 2014.

[24] S. De Ridder and E. Mooij, "Optimal terminal-area strategies and energy-tube concept for a winged re-entry vehicle," in Proceedings of the AIAA Guidance, Navigation, and Control Conference, AIAA Paper no. 2009-5769, Chicago, Ill, USA, August 2009.

[25] S. De Ridder and E. Mooij, "Optimal longitudinal trajectories for reusable space vehicles in the terminal area," Journal of Spacecraft and Rockets, vol. 48, no. 4, pp. 642-653, 2011.

[26] A. V. Rao, D. Benson, C. L. Darby et al., User's Manual for GPOPS Version 5.0: A MATLAB Software for Solving Multiple-Phase Optimal Control Problems Using hp-Adaptive Pseudospectral Methods, University of Florida, 2011. 

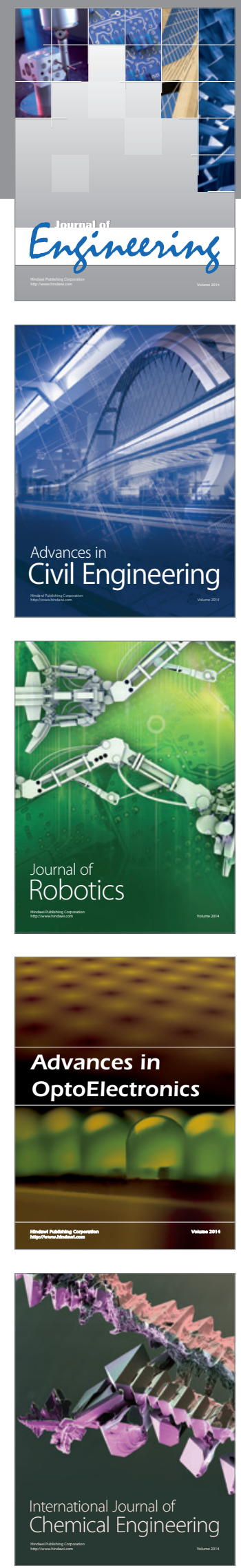

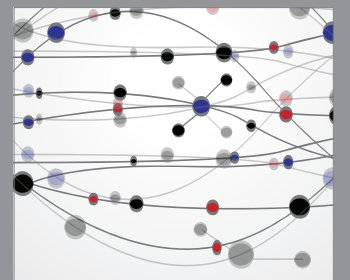

The Scientific World Journal
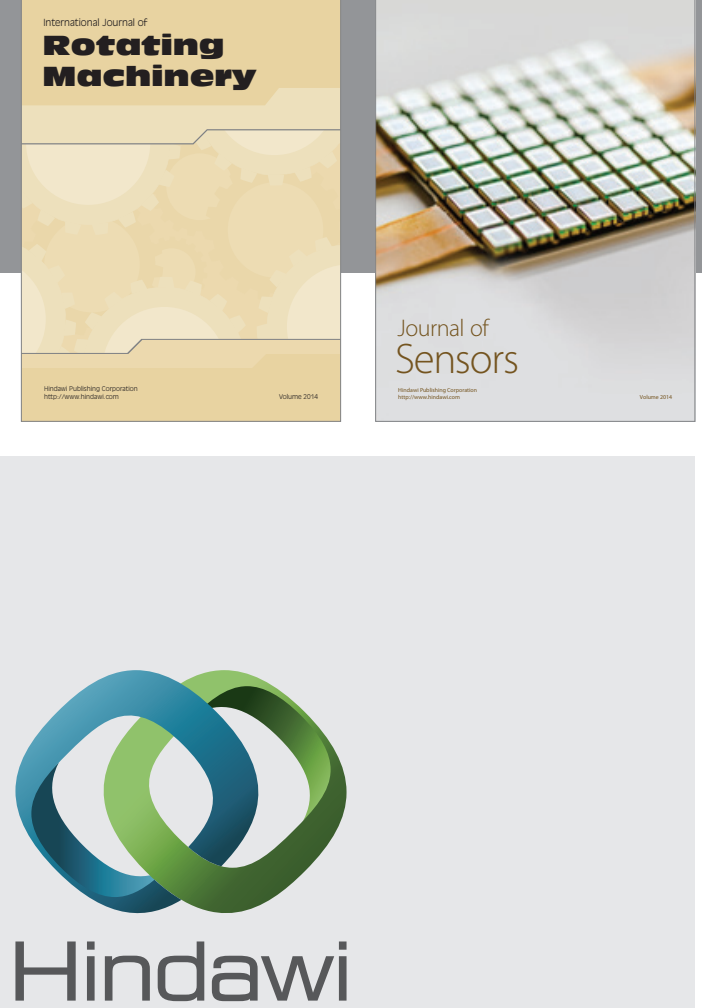

Submit your manuscripts at http://www.hindawi.com
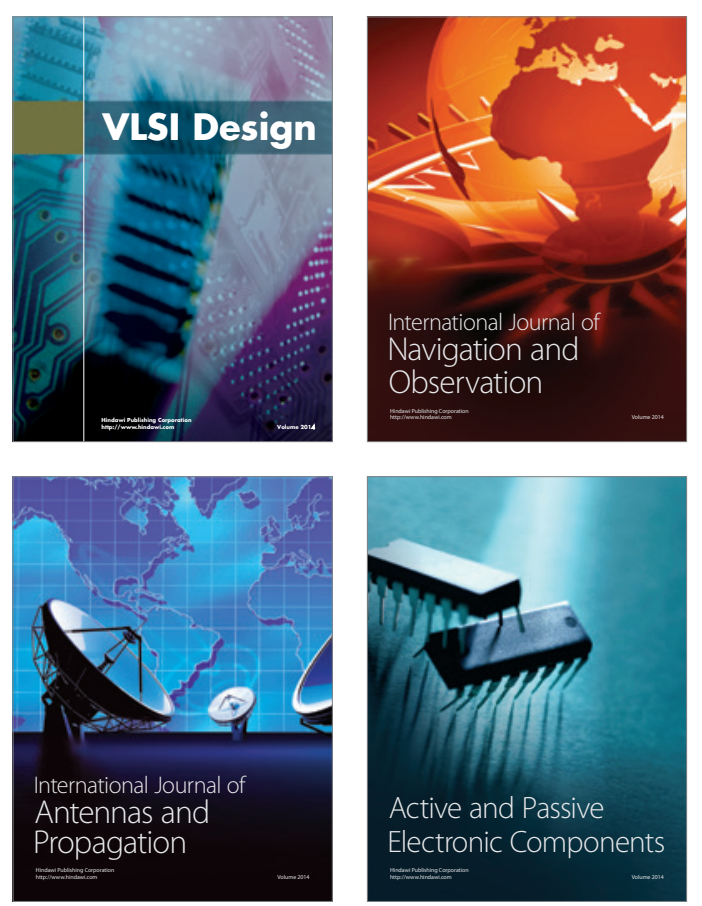
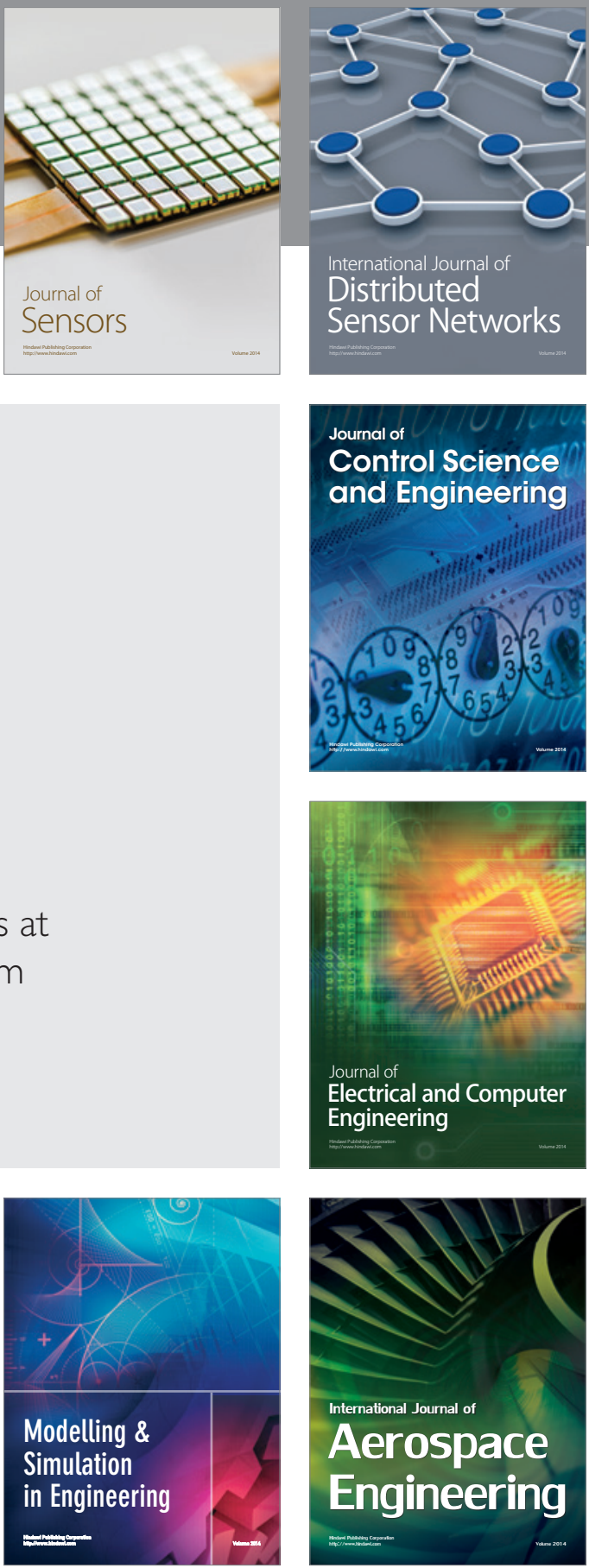

Journal of

Control Science

and Engineering
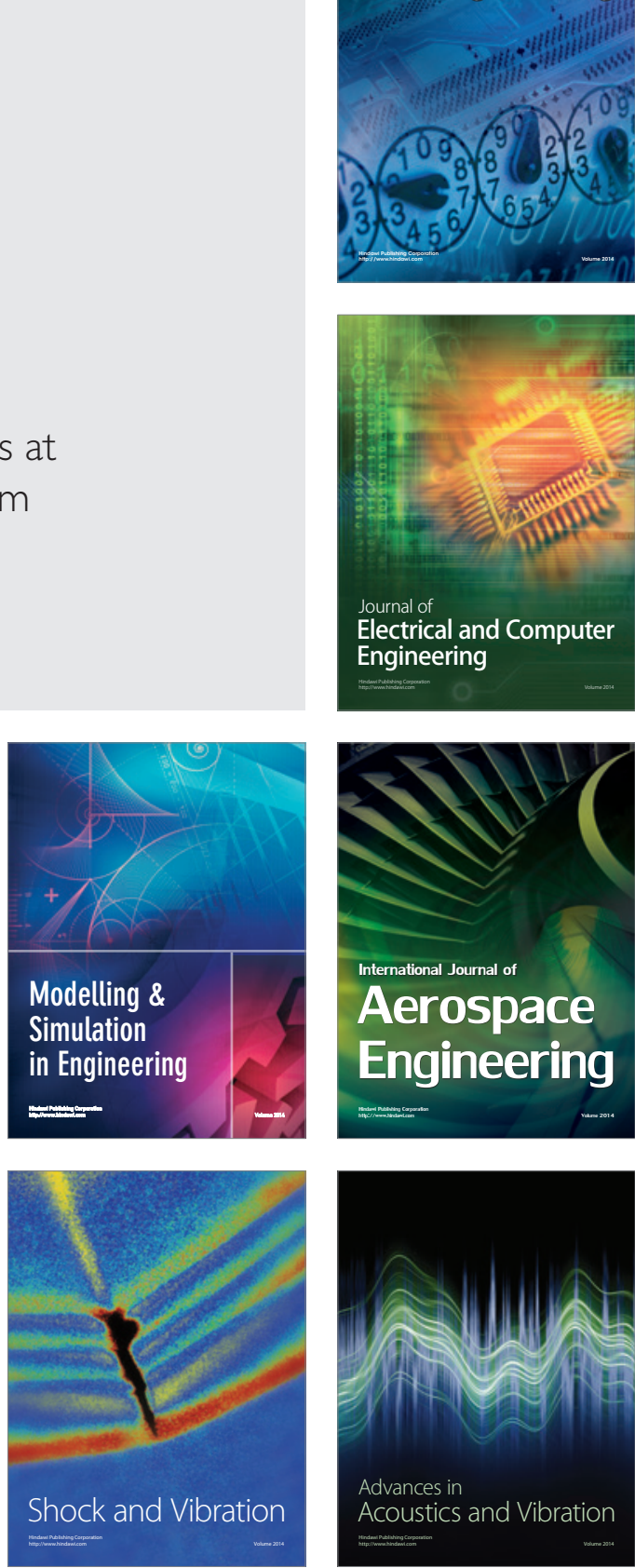